\title{
Selection from perceptual and conceptual representations
}

\author{
IRENE P. KAN and SHARON L. THOMPSON-SCHILL \\ University of Pennsylvania, Philadelphia, Pennsylvania
}

\begin{abstract}
The lateral prefrontal cortex has been implicated in a wide variety of functions that guide our behavior, and one such candidate function is selection. Selection mechanisms have been described in several domains spanning different stages of processing, from visual attention to response execution. Here, we consider two such mechanisms: selecting relevant information from the perceptual world (e.g., visual selective attention) and selecting relevant information from conceptual representations (e.g., selecting a specific attribute about an object from long-term memory). Although the mechanisms involved in visual selective attention have been well characterized, much less is known about the latter case of selection. In this article, we review the relevant literature from the attention domain as a springboard to understanding the mechanisms involved in conceptual selection.
\end{abstract}

Decades of research by Patricia Goldman-Rakic have shaped our current understanding of the functional organization of the primate prefrontal cortex (PFC). Combining anatomical, electrophysiological, neuropsychological, and neuroimaging data from human and nonhuman primates, Goldman-Rakic's scientific legacy includes a description of the PFC as a series of domain-specific, but computationally similar, processors that participate in networks consisting of sensory, motor, and limbic areas. Although the details of her arguments will continue to be debated, the notion that the PFC supports a computational mechanism that operates across a variety of domains has penetrated all the current research in this area. In this review, we will examine evidence about the role of the PFC in the selection of information across two broadly construed domains - perceptual and conceptual representations.

At any given moment, we are bombarded with a wide array of sensory information, from light hitting our retina to the sounds of dogs barking in the hallway. How do we filter out irrelevant information and focus on the task at hand? What are some of the factors that may affect our ability to filter out irrelevant information? What are the consequences of selectively focusing on some information and not on other? Active investigation of these and related questions over the last half-century has offered some possible solutions. In this article, we will briefly review

Funding was provided by grants from the National Institutes of Health (NIH R01 MH 60414 and NIH R01 MH 67008) and the Searle Scholars Program to S.L.T.-S. and by an NIH Predoctoral Training Grant in Behavioral and Cognitive Neuroscience to I.P.K. We thank Branch Coslett and Amishi Jha for helpful discussions and two anonymous reviewers for comments on an earlier draft of the manuscript. Correspondence concerning this article should be addressed to I. P. Kan, Department of Psychology, University of Pennsylvania, 3720 Walnut Street, Philadelphia, PA 19104-6241 (e-mail: ikan@psych.upenn.edu). one of the more prominent theories of visual selective attention and experimental evidence consistent with that theory (see Logan, 2004, for an extensive treatment of formal theories; see Luck \& Vecera, 2002, for a recent review of experimental evidence). We then will address some similar issues in another domain of selectionnamely, selection from among competing conceptual representations. Finally, we will conclude with a discussion of the possible mechanisms and neural circuitry that support selection from conceptual representations.

\section{VISUAL SELECTIVE ATTENTION}

Consider the popular children's game "Where's Waldo?" In this game, one's task is to find Waldo in a crowded environment, such as an amusement park. This game is sometimes considered to be challenging because there exist many distracting elements in the picture, including many Waldo look-alikes. In order to find the real Waldo, one must attend to task-relevant information and ignore other irrelevant information in the visual scene, and one may also use existing information to help guide the search. For example, one may already know that Waldo always wears a red-and-white striped shirt and carries a cane. During visual search, items that are consistent with this description are likely to attract one's attention, and items that are inconsistent with this description are likely to be ignored (e.g., people wearing blue shirts).

The mechanism involved in filtering out irrelevant information is often called selection. In the case of visual attention, selection occurs whenever one must choose among competing visual inputs. But why is it essential to filter out unwanted information? Numerous psychological studies have indicated that our cognitive system has a limited capacity and that only a finite number of items can be processed simultaneously. In other words, our ability to process information is restricted by our limited 
pool of cognitive resources (e.g., Kahneman, 1973). In order to conserve resources, we must allocate resources only to those processes that are most relevant to the current goal. Presumably, the more resources dedicated to a single process, the more efficiently the process can be accomplished (i.e., it is faster and more accurate). On the other hand, as the number of items demanding cognitive resources increases, the resource available for each item decreases, and search efficiency declines. Therefore, both the number of items demanding resources and the amount of resources each item requires can affect informationprocessing efficiency.

For example, when presented with an array of letters visually, subjects are faster at identifying a target as the number of relevant distractors decreases (e.g., Bundesen, 1990, 1996; Egeth, Virzi, \& Garbart, 1984). When shown an array of green and red letters, subjects' efficiency at detecting a target (e.g., a red "A") will be affected by the number of relevant distractors (i.e., red letters), but not by the number of irrelevant distractors (i.e., green letters). This is because subjects are successful at attending to relevant information (i.e., red letters) and ignoring irrelevant information (i.e., green letters).

Physical limitations of our visual system also contribute to competition and, thus, the necessity for selection. For example, when monkeys are shown a preferred stimulus (i.e., a stimulus that elicits a high firing rate) and a nonpreferred stimulus (i.e., a stimulus that elicits a low firing rate) within the same receptive field, the neuron's response is the weighted average of the responses to the two individual stimuli. This observation has been interpreted as the two stimuli's interacting in a mutually suppressive way, and this effect is termed the sensory suppression effect. This finding suggests that the two stimuli within the same receptive field are processed collectively and that the smallest visual unit that can be processed independently is limited by the size of the receptive field (e.g., Moran \& Desimone, 1985).

Kastner, De Weerd, Desimone, and Ungerleider (1998) used functional magnetic resonance imaging (fMRI) to investigate the possible role of sensory suppression in humans. On each trial, subjects were presented with four colorful, abstract, and complex images, either sequentially or simultaneously. In the sequential condition, each image was shown in one of four locations, and in the simultaneous condition, all four images were shown in all four locations at the same time (see Figure 1). If the human visual system were to exhibit sensory suppression effects, the average response integrated over time for the sequential condition should be larger than the response for the simultaneous condition, because suppression is expected to occur only when stimuli are presented simultaneously.

Not only did Kastner et al. (1998) observe a sensory suppression effect in their subjects, they also reported that the sensory suppression effect was scaled to the size of the neuron's receptive field. Specifically, when compared with the sequential condition, activation in V1 was similar in the simultaneous condition (i.e., no suppression effect), activation in V2 was reduced in the simultaneous condition (i.e., small suppression effect), and the reduction of the activation level in the simultaneous condition was even greater in V4 and TEO (i.e., large suppression effect). The authors attributed this pattern of data to the difference in the size of the receptive field for neurons in these regions. Because receptive fields in V1 and V2 are small, they would encompass only a small portion of the display (see Figure 1); however, since receptive fields in V4 and TEO are larger, they would encompass the entire display. That is, the more items a receptive field encompasses, the higher the level of competition, and the larger the suppression effect. In a follow-up experiment, when the spatial separation between the stimuli was increased, the suppression effect in V4 and TEO diminished, confirming that the suppression effect is scaled by the size of the receptive field and by the number of items enveloped within it (Kastner et al., 2001).

Given the limitations of our cognitive system (i.e., limited capacity) and of our visual system (i.e., receptive field size limitation), Desimone and Duncan (1995) proposed a biased competition model of visual selective attention. The authors proposed that when competition arises, the attentional system's role is to bias our selection process by considering both bottom-up and topdown influences. In addition, there exists an attentional template, which contains a short-term description of information that is currently task relevant (e.g., goal-grab a ripe banana), and information in this template is used to bias selection. A perceptual stimulus is compared with the attentional template, and the greater the similarity between the perceptual stimulus and the attentional template, the more bias the stimulus receives. The more bias the stimulus gets, the higher its competitive weight and the stronger the inhibitory influence it has over the competing alternatives. Furthermore, the attentional template is described as a flexible template that can represent any property (e.g., location, shape, or color) necessary to facilitate selection. Initial competition occurs within each domain represented in the template (e.g., spatial-on the left, shape-the long thing, or color-the yellow thing), and a winner from one domain will exert an inhibitory influence over other domains. In other words, inhibitory effects can be found both within domain and across domain, and accumulation of evidence is thought to occur in parallel. Finally, a winner emerges as a result of converging evidence from across different domains (see also Driver \& Frackowiak, 2001; Kastner \& Ungerleider, 2000).

Consider three items on your kitchen counter-a yellow banana, a green banana, and a yellow lemon. If your goal is to grab a ripe banana to make banana bread, your attentional template may include a representation of ripe banana, which may in turn include color and shape information. As was suggested earlier, in order to resolve competition, information from each domain (i.e., color and shape) contributes to the selection process by updating the competitive weight of each stimulus. Therefore, in the 


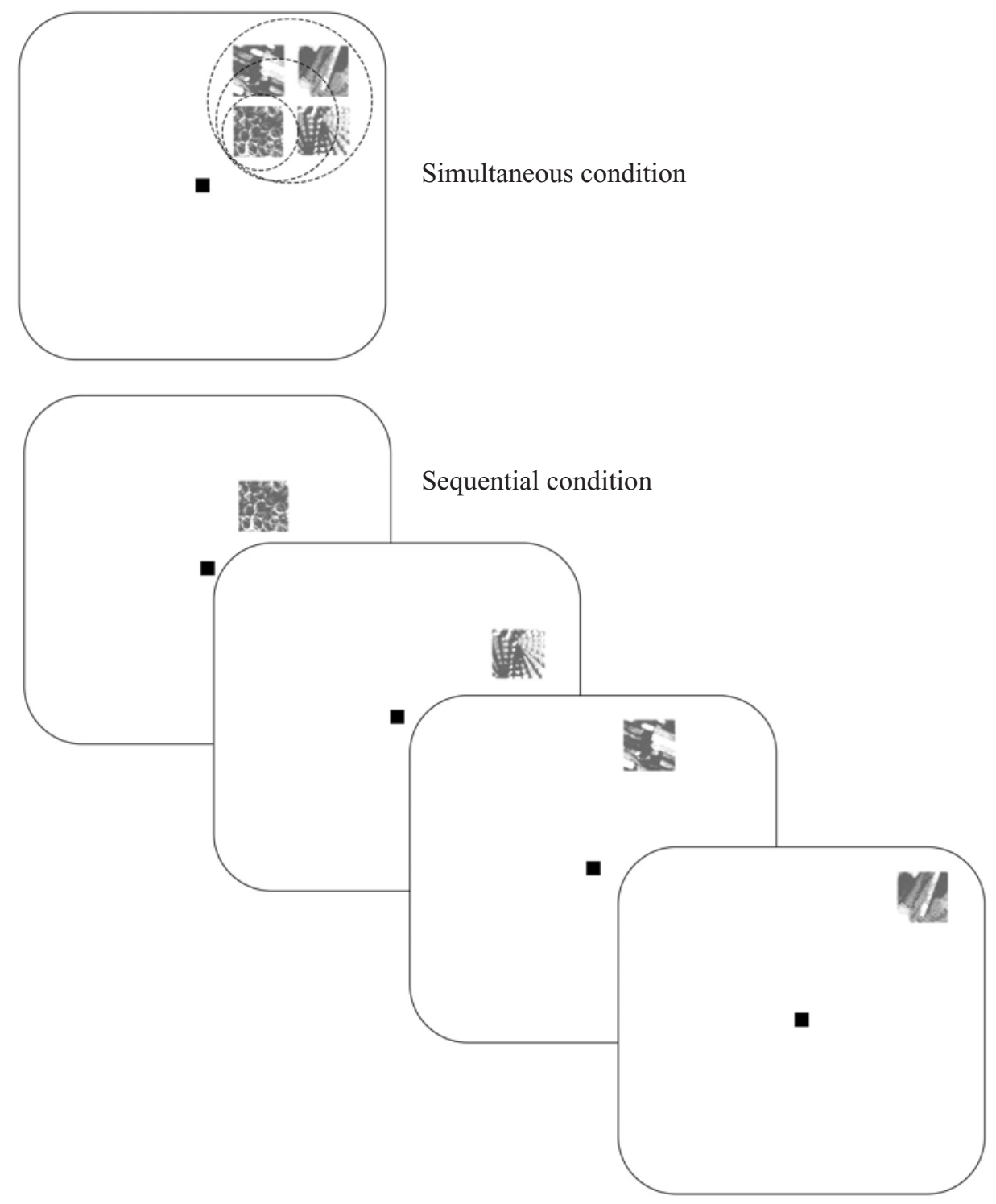

Figure 1. Complex images used in Kastner, De Weerd, Desimone, and Ungerleider's (1998) fMRI experiment (original stimuli in color) — simultaneous condition (top panel) and sequential condition (bottom four panels). Dotted circles represent hypothetical sizes of receptive fields in different extrastriate regions-V1 (inner circle), V2 (middle circle), V4 and TEO (outer circle).

color domain, the yellow banana and the yellow lemon will receive a boost in their competitive weights, and as a result of inhibition from the yellow items, the green banana's competitive weight will decrease. In the shape domain, however, both bananas will receive an increase in competitive weights, and the lemon's competitive weight will decrease. Cumulative evidence across the color and shape domains will lead to selection of the yellow banana as the winner, and it will be selected for further processing.

\section{Neural Circuitry of Selective Attention}

Numerous neuropsychological and neuroimaging studies have provided evidence that visual selective attention involves a distributed network of primary visual areas and higher order areas in the frontal and parietal cortices. Whereas domain-specific competition is thought to occur locally within domain-selective regions, across-domain competition is partially resolved by attentional-biasing mechanisms, which are thought to be mediated by a frontoparietal circuit (see Kastner \& Ungerleider, 2001, and Pessoa, Kastner, \& Ungerleider, 2003, for reviews; see also Sylvester et al., 2003, for a recent proposal of the parietal cortex's role in attentional allocation and attention switching). For example, color-selective competition may occur in V4, motion-selective competition may take place in V5, and spatial location selection may occur in 
the parietal cortex. The fronto-parietal network biases information by exerting inhibitory influence over competitors. It has also been suggested that another brain region, the lateral PFC, plays a critical role in guiding behavior by incorporating bottom-up and top-down influences (see Fletcher \& Henson, 2001, and E. K. Miller \& Cohen, 2001 , for recent reviews). In addition, the attentional template's reliance on working memory also suggests the possible involvement of the lateral PFC (e.g., Desimone \& Duncan, 1995; Fuster, 1973; Fuster \& Alexander, 1971; Goldman-Rakic, 1987; Jacobsen, 1935, 1936).

\section{What Are Some Factors That Affect Selection?}

A wide variety of factors affecting selection efficiency have been identified, and they can be roughly divided into two classes - bottom-up and top-down influences. Bottomup influences refer to qualities that are inherent to the stimuli themselves (e.g., size, color, or brightness), and topdown influences refer to factors that are manipulated by task instructions or current context. Here, we will briefly review several bottom-up and top-down factors that have been shown to influence visual selective attention (see Luck \& Vecera, 2002, for a comprehensive review).

Bottom-up influence: Local homogeneity. Returning to finding Waldo, if everyone else in Waldo's world wears black-and-white clothing, finding Waldo will not be much of a challenge. This phenomenon, called popout, refers to the situation in which a unique target in an otherwise homogeneous field pops out from the visual scene (e.g., Green \& Anderson, 1956; Treisman \& Gelade, 1980). In this situation, as long as the distractors are homogeneous, the number of distractors present in the field has little effect on search efficiency (Figure 2A). On the other hand, in a display with a heterogeneous field, the number of distractors has a strong influence over search efficiency (see Figure 2B).

Another way to manipulate homogeneity is by varying the physical size of the stimuli. For example, Figures 2B and $2 \mathrm{C}$ consist of the same elements with heterogeneous shapes, but searching for the target (i.e., a circle) in Figure $2 \mathrm{C}$ will be much easier than searching in Figure 2B. Since the physical size of the circle is disproportionately larger in Figure 2C, a pop-out effect is expected: The uniquely large item pops out from a field of homogeneous smaller items. In addition to size manipulation, Treisman and Gormican (1988) reported that heterogeneity in other perceptual features, such as brightness contrast and line length, yielded similar results.

In sum, these studies suggest that the uniqueness of the target may play a role in visual selective selection. In a recent study, Pashler and Harris (2001) reported that a unique item is better detected and also better remembered. In one experiment, subjects participated in a single trial, in which they were shown an array of six words for a brief time and were asked to describe what they saw. For some subjects, one of the items flashed on the screen while the others remained static, and for other subjects, one of the items remained static while the others flashed on the screen. Regardless of feature status (i.e., flashing vs. static), the probability of recalling a unique item was significantly higher than the probability of recalling a nonunique item. Furthermore, the magnitude of the uniqueness advantage was greater for flashing items than for static items. This pattern of results was

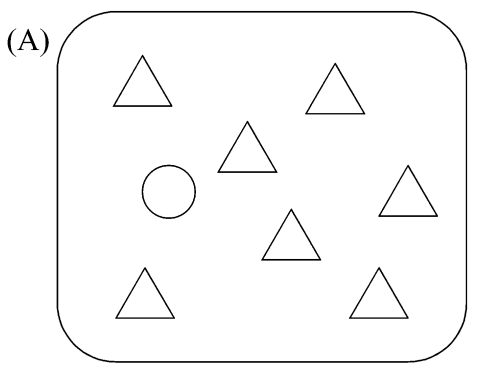

(B)
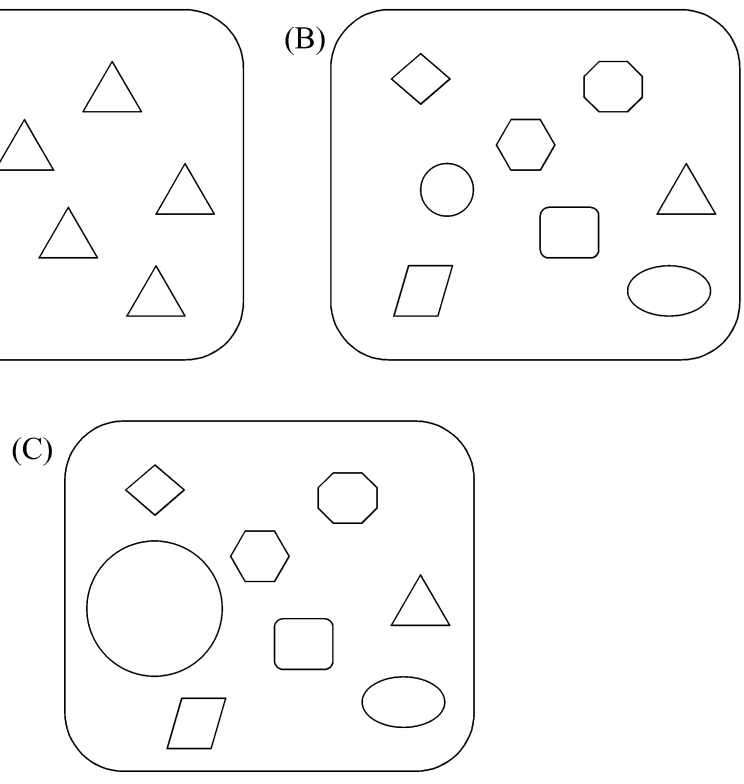

Figure 2. Visual search displays. Searching for a target (i.e., a circle) in (A) a homogeneous scene, (B) a heterogeneous scene, or (C) a display with heterogeneous elements and distractors of homogeneous size. 
replicated in a second experiment, in which line drawings and aesthetic judgments were used. Taken together, these results suggest that both the uniqueness of the target and the inherent quality (e.g., flashing vs. static) of the target have an effect on selective attention.

Bottom-up influence: Abrupt stimulus onset/offset. It has also been reported that sudden onset of a stimulus can capture attention. The argument is that it is evolutionarily advantageous for us to pay attention to a sudden onset of a stimulus, because that may indicate the emergence of a predator (Luck \& Vecera, 2002). Yantis and Jonides (1984) investigated the effect of abrupt stimulus onset with a visual search task in the laboratory. Two trial conditions were included, an onset condition and an offset condition, which defined how the target was presented in the search display. Across both trial types, the subjects were always shown a display with three "figureeight placeholders" at the beginning of each trial (see Figure 3, top panel), and they were asked to fixate at the circle in the center. To create the search display, some of the lines of each placeholder were removed to create a letter (e.g., removing the two right vertical lines to create the letter "E"). Furthermore, a new letter was introduced simultaneously in the previously unoccupied location (see Figure 3, bottom panels). In the example illustrated in Figure 3, the subject's task was to detect the target letter " $A$ " in the search display, and they were told that the target was equally likely to appear in any of the four locations. Therefore, the subjects had no incentive to selectively attend to any of the four locations prior to stimulus presentation.
If the subjects' attention were to be captured by abrupt onset, enhanced performance would be expected if the target appeared in the previously unoccupied location (i.e., onset condition). Furthermore, impaired performance would be expected if the target appeared elsewhere in the display, because the subjects must disengage their attention from the location of the abrupt onset and begin to search for the target in the other locations (i.e., offset condition). On the other hand, if abrupt onset did not capture attention, similar performances would be expected across all the locations. Indeed, Yantis and Jonides (1984) found that subjects were faster at detecting a target when it appeared in the previously unoccupied location (i.e., the onset condition) than when it appeared in a previously occupied location (i.e., the offset condition). These data support the idea that abrupt onset of a stimulus captures attention.

Although Yantis and Jonides (1984) did not report attentional capture as a result of stimulus offset, J. Miller (1989) argued that Yantis and Jonides's results may be explained by the possibility that stimulus onset may be more perceptually salient than stimulus offset. Thus, previously reported behavioral differences between abrupt onset and offset may reflect a difference in perceptual salience, rather than inherent differences between abrupt onset and offset. To test this idea, J. Miller varied the number of onset features and offset features and found that attention was captured by the greatest amount of overall display change, even when the change involved offsets only.

Top-down influence: Task set. Attentional capture in the face of abrupt stimulus onset is generally con-
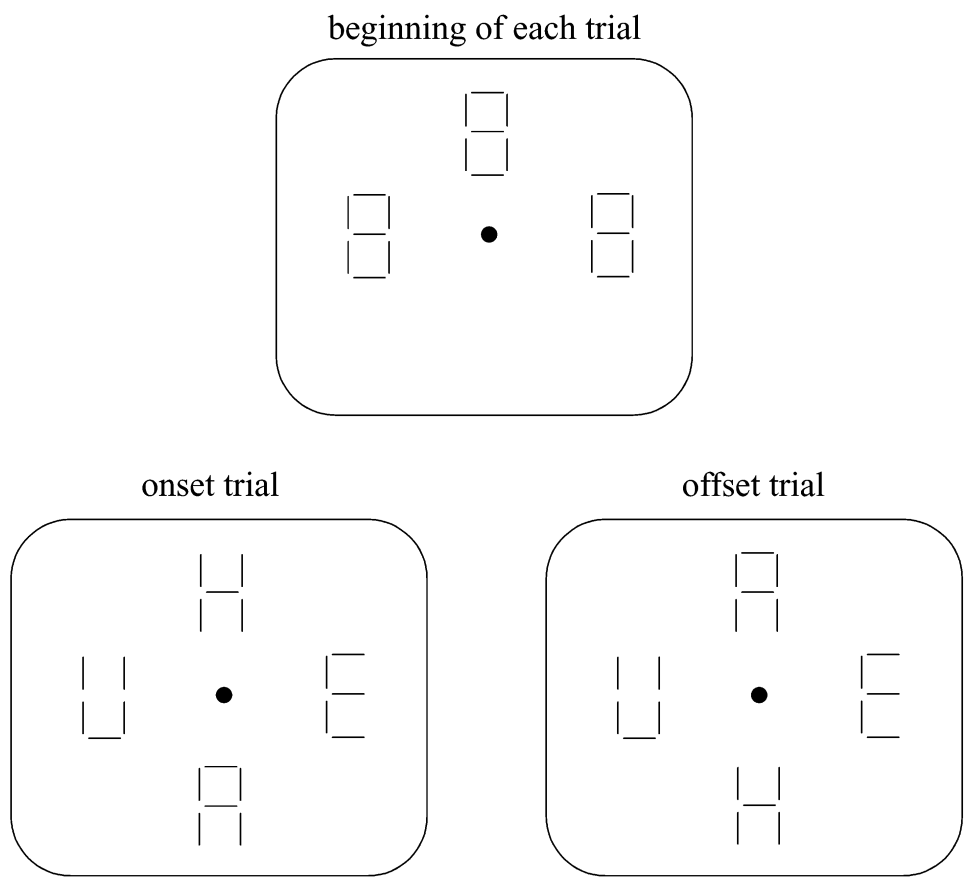

Figure 3. Visual search displays: examples of stimuli used in Yantis and Jonides's (1984) visual search experiment. 
ceived of as involuntary, and it is thought that attentional capture occurs as a result of the intrinsic properties of the onset (i.e., the sudden change in stimulus features). However, recent theorizing has questioned that interpretation. Specifically, Folk and colleagues (Folk \& Remington, 1999; Folk, Remington, \& Johnston, 1992; Folk, Remington, \& Wright, 1994) proposed that abrupt onset of a stimulus does not have priority access to the attentional system per se, but that attentional capture is contingent upon the relationship between the features of the event that capture attention and the features of the target that are task relevant. In other words, attention is most likely to be captured if there is a large overlap between the features that define the onset and the features that define the target. They argued that subjects might have adopted a task set of "onset detection" because onset might also indicate the presence of a target. However, when onset does not predict the presence of a target, abrupt onset is not expected to capture attention. This proposal is called the contingent involuntary orienting hypothesis.

In a series of experiments, Folk et al. (1992) manipulated feature overlap between the onset and the target in a spatial-cuing task. In general, in a spatial-cuing task, subjects are presented with a cue to one of many possible spatial locations, and the probability of the cue's being valid (i.e., a target appearing in the cued location) varies from experiment to experiment. In Folk et al.'s (1992) experiments, targets were either characterized by an abrupt onset (e.g., an " $\mathrm{X}$ " appearing in a previously unoccupied location) or signaled by a color change (e.g., a white " $X$ " changing into a red "X"). Furthermore, cues were characterized either by abrupt onset of small circles surrounding one of the spatial locations or by a color change in the small circles surrounding one of the four
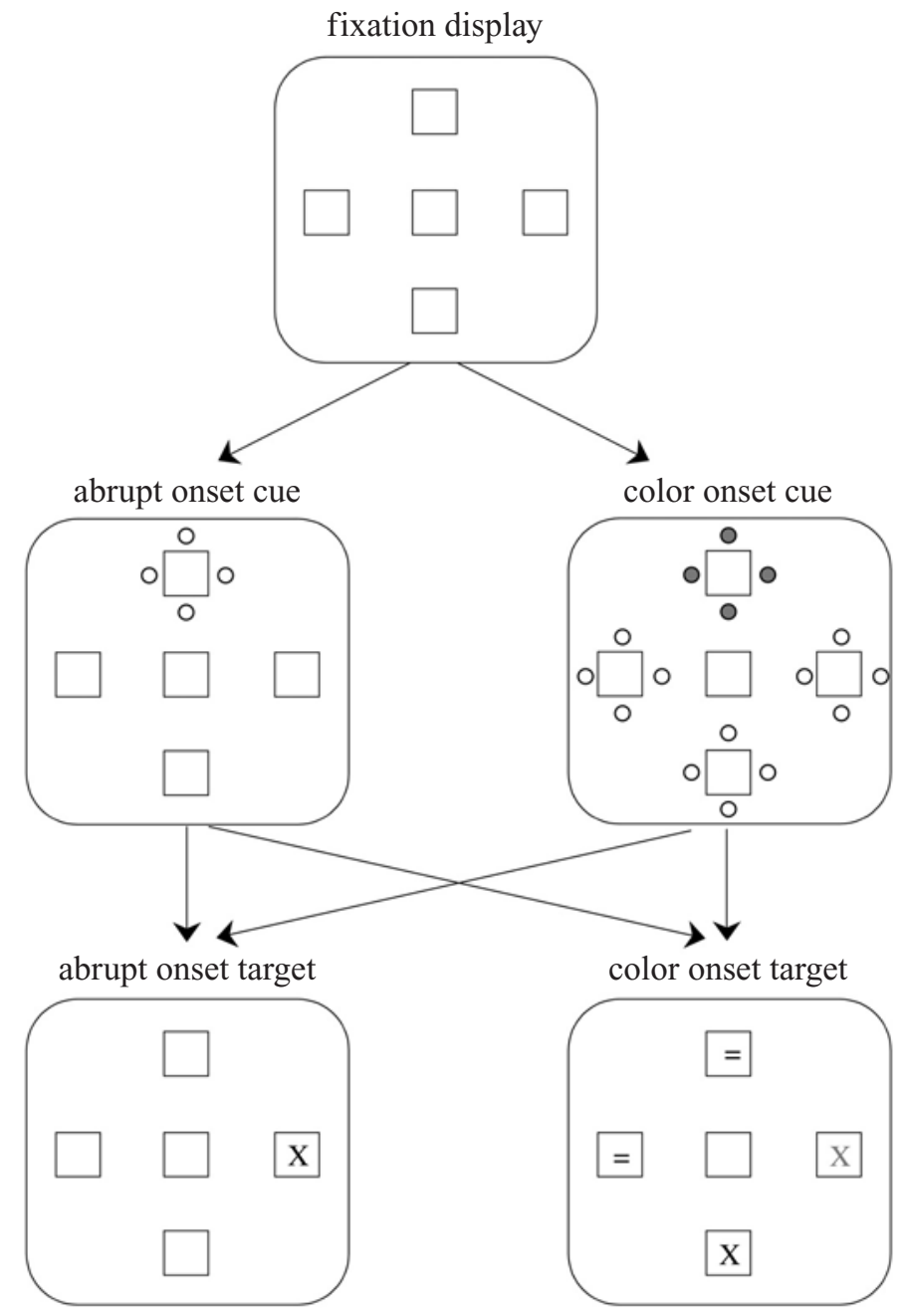

Figure 4. Spatial-cuing paradigm used in Folk, Remington, and Johnston's (1992) experiments. The colored dots and the colored $X$ have been changed to solid gray dots and a gray $X$ for the purpose of this illustration. 
locations (see Figure 4). The authors demonstrated that attention was captured only when the cue and the target shared the same critical feature (i.e., abrupt onset or color onset). In other words, attentional capture was elicited by a task-relevant feature (i.e., a feature within the task set), not by mere abrupt onset of the stimuli. This pattern of results suggests an important role for topdown influence in attentional capture.

Top-down influence: Task instructions. What are some other ways in which task instructions influence selective visual attention? How might changing the task from "Where's Waldo?" to "Where's the clown?" affect performance? With these new instructions, it is likely that an individual's attentional template will change from red-and-white striped shirt to giant shoes. As a result, the attentional system may bias shape and size templates, instead of the color template. Task instructions may also affect the status of potential competitors. For example, under the Waldo instructions, other characters wearing red-and-white striped shirts are potential competitors and will affect selection, but under the clown instructions, these same characters are no longer task relevant and are unlikely to exert influence over search efficiency. In other words, an item's status as a competitor can be changed as a result of task instructions.

In a visual search experiment, Jonides and Gleitman (1972) explored the effects of category membership on visual search efficiency. In a series of behavioral experiments, the researchers found that subjects were slower at visual search when the target and the distractors belonged to the same category (i.e., letters) than when the target and the distractors belonged to different categories (i.e., letter target and digit distractors). In one condition, category membership of an ambiguous stimulus ("O”) was determined by top-down task instructions, not by its physical characteristics. The subjects were to detect the target "O" either in a field of letters or in a field of digits. In the same-category conditions, the target "O" was introduced as the letter "oh" (letter-letter condition) or as the number zero (number-number condition), and in the mixed-category condition, the target was introduced either as a letter in a field of digits or as a digit in a field of letters. The category effect demonstrated that local homogeneity of a visual scene could be manipulated by top-down influence as well.

In a similar experiment, Hamm and Taylor (1997) used a rapid serial visual presentation (RSVP) paradigm to explore the same question. In an RSVP task, subjects are presented with a series of items at a very rapid rate (e.g., ranging from 6 to 20 items per second) and are asked to identify a target and to detect a probe within the stream of stimuli. A classic phenomenon associated with the RSVP paradigm is that subjects' ability to detect a probe on trial $n$ is impaired if they were successful at identifying a target on trial $n-1$. In other words, subjects typically suffer a cost at probe detection if the probe appears immediately after a target and if the target is correctly identified.
Hamm and Taylor (1997) explained this phenomenon as competition from members within the same category. In most experiments in which probe detection interference has been reported, items from the same category were used for both the probes and the targets (e.g., letter target and letter probe or number target and number probe). Since the items are presented at such a rapid rate, it is likely that the cognitive system has not yet completed processing of the target when the probe is presented. Therefore, the probe is left undetected because the system is preoccupied. To test this idea, the authors proposed that the probe detection interference effect should be attenuated if the probe and the target are processed under different cognitive processors. They suggested that items from different categories, such as letters and numbers, would present such a situation, assuming that items from different categories may be processed in separate processors.

In one of their experiments, Hamm and Taylor (1997) manipulated the categorical relationship between the target and the probe. Whereas the target was always a letter printed in white, the probe was always an "O" printed in black. The subjects were asked to identify the white letter and to detect the black "O." The authors manipulated the category membership of the "O" stimulus by telling the subjects that the probe was either the letter "oh" or the number "zero." Therefore, the authors were able to use the same stimulus but still were able to manipulate stimulus category with top-down processes. A probe detection deficit was found in the same-category condition, but not in the different-category condition. That is, the subjects were impaired at detecting the probe when the target and the probe shared category membership (i.e., letter-letter) but not when the probe and the target were from different categories (i.e., letter-number). These data demonstrated the importance of top-down influence over selective attention. In the letter-number condition, the task instructions biased the attentional system toward number detection. Thus, number stimuli received higher weights and exerted inhibitory influence over nonnumber stimuli.

Lexical category has also been shown to affect attentional processes. Using the RSVP paradigm, Staller (1982) presented subjects with a series of letter strings, and the subjects were asked to detect a precued word (e.g., chair) or an anagram of the precued word (e.g., raich). Staller found a reliable word superiority effect in detection performance. That is, the subjects were reliably better at detecting the precued words than at detecting their anagrams. This is evidence suggesting that familiarity and long-term memory play a role in visual selective attention. In the context of the biased competition model, it is likely that familiar items (i.e., real words) have higher initial competitive weights than do unfamiliar items (i.e., nonwords). Thus, the attentional system may be biased toward selecting the word stimuli.

Chawla, Rees, and Friston (1999) explored the effect of task instructions on neural activity in extrastriate vi- 
sual areas. Subjects were presented with a monochromatic and stationary random-dot display. The subjects were also told that the dots in the display would change intermittently to red dots and that the red dots would move radially. On some trials, pink dots might appear among the red dots, and some dots might also appear to move more slowly than other dots. To bias a color attentional set, the subjects were told to detect the presence of pink dots among the red dots in the display, and to bias a motion attentional set, the subjects were told to discriminate a set of dots that moved more slowly from among a set of dots that moved more quickly.

The authors reported enhanced baseline activity in areas V4 (color area) and V5 (motion area) in response to color attentional set and motion attentional set, respectively. Importantly, the enhanced baseline activity was observed even when the color or motion information was absent from the stimulus (see Buchel et al., 1998, Kastner, Pinsk, De Weerd, Desimone, \& Ungerleider, 1999, and O'Craven, Rosen, Kwong, Treisman, \& Savoy, 1997, for similar findings). In other words, early perceptual areas are primed by the attentional set for subsequent processing of task-relevant information. These data are consistent with the biased competition model, in that the attentional system is biased toward a specific attribute (i.e., color or motion), and brain regions selective for those domains are primed for domain-selective processing by enhancing baseline activity.

Summary. In this section, we described the influence of bottom-up and top-down factors on visual selective attention. To summarize, (1) when an item is selected for further processing, performance is enhanced, (2) unique items are more likely to be selected for further processing than nonunique items are, (3) attention can be captured by sudden stimulus onset or offset, (4) effects of bottom-up factors may be further modulated by topdown processes, such as task set, (5) task set prepares early visual areas for a specific type of stimulus, (6) longterm knowledge may affect interpretation of the same physical stimulus as a target or as a competitor, and (7) long-term memory may also bias our attention toward familiar items.

\section{FINDING THE INTERNAL WALDO Selection From Conceptual Representations}

In this section, we will address some issues that pertain to selection among competing conceptual representations. We will use the term conceptual to refer to learned, stable representations of information that is not necessarily immediately present in the environment. This can be contrasted with perceptual representations of stimuli that are transiently present in the external environment. Although the distinction between perceptual and conceptual representations is likely to be fuzzy (and perhaps is best thought of as a continuum; e.g., representations of occluded stimuli in the environment), for our present purposes, we will treat the perceptual-conceptual distinction as a dichotomy.

Why is it necessary to select among conceptual representations? How is selection accomplished? What are the consequences of failure to select among competing alternatives? Throughout this discussion, we will draw parallels to the literature on visual selective attention when possible, to highlight some of the potential similarities between the mechanisms involved in perceptual and conceptual selection. Note that we are not arguing here that a common mechanism subserves both types of selection. As has been suggested by previous research, generalizing theoretical models developed in one domain to another warrants special caution (e.g., Farah, 1989), and the extent of overlap between the two domains of selection described here remains an empirical question. In contrast, our goal is to explore how ideas that have emerged from theoretical and empirical work in the domain of visual selective attention may inform our understanding of selection among competing conceptual representations.

\section{An Example of Selection Failure}

Perhaps one of the most striking examples of selection failure is utilization behavior, as exhibited by braindamaged patients. Utilization behavior is characterized by stimulus-driven behaviors, regardless of the appropriateness of the action given the situation. For example,

[French neurologist Lhermitte] would invite a patient to a meeting and place a hammer, nail, and picture on a table in the entryway. When encountered by this array of objects, a frontal patient might pick up the hammer and nail and hang the picture on the wall. In another instance, Lhermitte put a hypodermic needle on his desk, dropped his trousers, and turned his back to the patient.... The patient was unfazed. He simply picked up the needle and gave his doctor a healthy jab in the buttocks! (Gazzaniga, Ivry, \& Mangun, 2002, p. 548)

In this anecdote, the patient's behavior was completely driven by the objects in front of him. When encountered with a hammer, a nail, and a picture, he automatically hung the picture on the wall, because the action most often associated with those objects is hang. This automatic execution of the prepotent response (i.e., the response most commonly associated with a given stimulus) can be likened to an internal pop-out effect. In the framework of visual selective attention, we discussed pop-out as an automatic response that is driven by the salience of the stimulus. The salient target is selected because its baseline competitive weight is the highest. A similar analogy can be applied to utilization behavior. Consider the hypothetical conceptual network illustrated in Figure 5. The action that is commonly activated by the three concepts is "hang," which suggests that "hang" is likely to have a high baseline competitive weight within this hypothetical network, because it is gaining bias from all three concepts. The patient's behavior is considered 


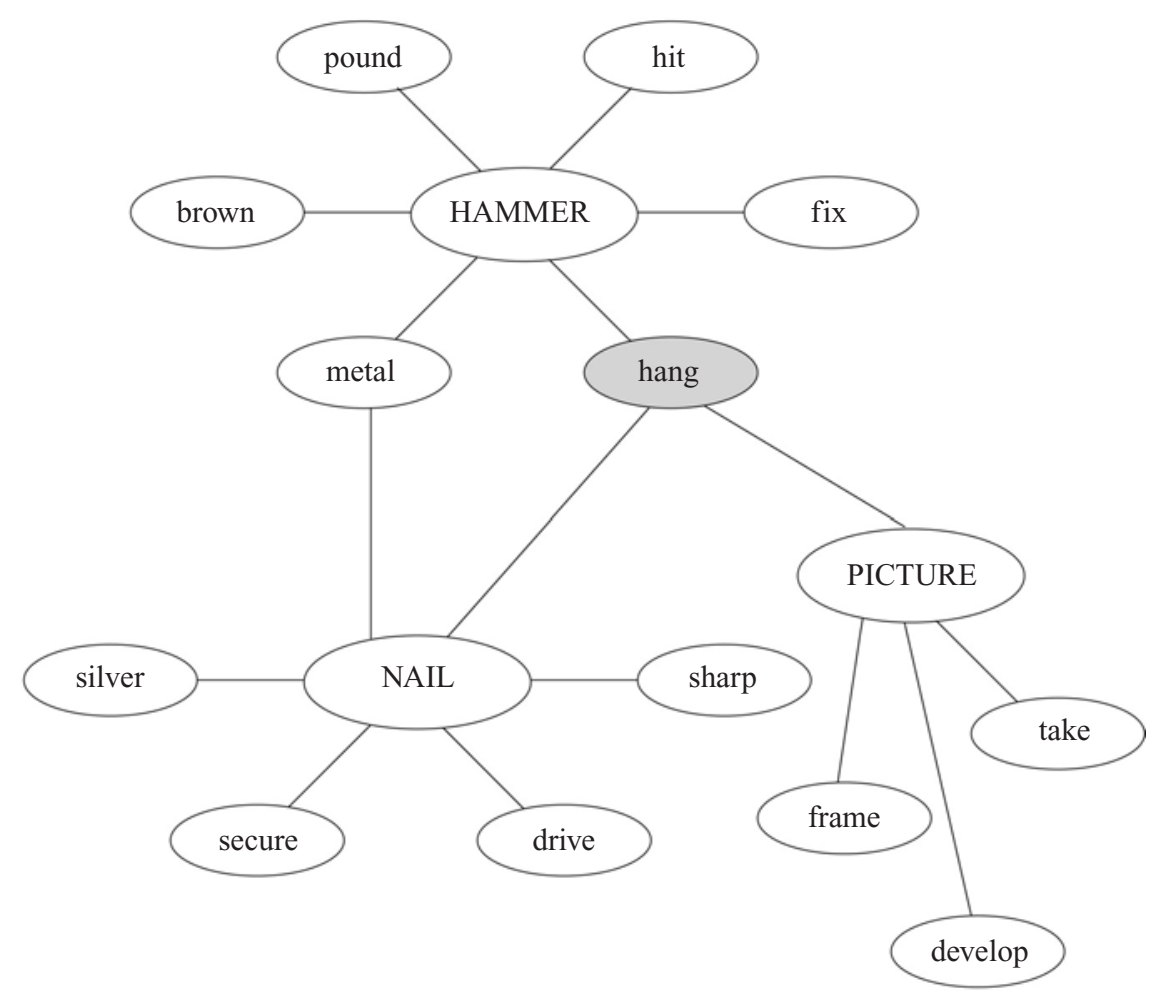

Figure 5. Hypothetical conceptual network for the concepts hammer, nail, and picture.

as a selection failure because he failed to select against the prepotent response. Given the context of the situation (i.e., someone else's home), his task set should have guided him to select against "hang." In other words, the patient was unable to inhibit an inappropriate representation, possibly due to a failure in the biasing mechanism.

\section{Neural Circuitry Involved in Conceptual Selection}

Inhibition of an inappropriate response is one of many facets of cognitive control. Cognitive control, a general guiding mechanism, allows us to adapt our behaviors to different situations and contexts, instead of behaving in a way that is completely driven by perceptual stimuli (e.g., utilization behavior). There are at least two anatomical regions linked to cognitive control - the anterior cingulate and the lateral PFC. Several investigators have suggested that the lateral PFC guides our behavior by incorporating top-down attentional bias and bottom-up influences (see Fletcher \& Henson, 2001, and E. K. Miller \& Cohen, 2001, for reviews). On the other hand, the anterior cingulate is thought to be associated with conflict detection and monitoring (e.g., Botvinick, Braver, Barch, Carter, \& Cohen, 2001).

To evaluate the functional dissociations between the lateral PFC and the anterior cingulate, using fMRI, Milham et al. (2001) measured neural activity during a color- word Stroop task. In the color-word Stroop task, subjects are presented with a color word (e.g., red) printed in either a congruent ink color (e.g., red ink) or an incongruent ink color (e.g., green ink), and the subjects' task is to ignore the identity of the word and to name the ink color (Stroop, 1935). The classic finding is that subjects are significantly slower at color naming on incongruent trials than on congruent trials, because of the conflict present in the incongruent stimulus. In addition to manipulating congruency, Milham and colleagues also manipulated the response eligibility of incongruent color words. Specifically, in the response-eligible condition, incongruent color words also named ink colors that are possible responses, and in the response-ineligible condition, incongruent color words do not name any of the possible ink colors. For example, if the possible ink colors are red, green, yellow, and blue, these four words are also used as incongruent color words on incongruent trials in the response-eligible condition; on the other hand, in the response-ineligible condition, these four words appear in the congruent condition only and are never used as incongruent color words. This manipulation is akin to the task set manipulation described in an earlier section (cf. the contingent orienting hypothesis in attentional capture).

Milham et al. (2001) reported that whereas the dorsolateral and posterior inferior PFCs are involved in selection of competing conceptual representations, the anterior cingulate is recruited only when conflict on a response 
level is present (see also Barch, Braver, Sabb, \& Noll, 2000; Nelson, Reuter-Lorenz, Sylvester, Jonides, \& Smith, 2003). Similarly, using a flanker task, van Veen, Cohen, Botvinick, Stenger, and Carter (2001) also reported that the anterior cingulate is selectively responsive to response conflict, and not to conflict on the level of conceptual representations. The authors suggested that this pattern of data may be related to the interconnectivity between the motor system and the anterior cingulate. Taken together, these data suggest that whereas the lateral PFC is involved in selection on a conceptual level, the anterior cingulate is involved in selection on a response level. In the present discussion, we will focus on selection among conceptual representations and the role of the lateral PFC in this process (see Duncan \& Owen, 2000, and Schumacher \& Jiang, 2003, for discussions on how response representations compete with each other).

\section{What Are Competing Conceptual Representations?}

In an earlier section, we described visual selective attention as the selection of relevant information from a cluttered perceptual environment. When selection from conceptual representations is discussed, it may be helpful to think of the mechanisms involved as selecting relevant information from the internal clutter. Similar to our discussion of visual selective attention, competition can occur on at least two levels: within domain and across domains. As is suggested by the biased competition model of selective attention (Desimone \& Duncan, 1995), initial competition occurs within each domain (e.g., color, shape, or location), and a winner from one domain exerts inhibitory influence over other domains. Subsequent competition across domains will yield a final winner. We propose that a similar mechanism may be at work in conceptual selection.

For example, consider the simple task of retrieving the color of an apple when the object is not immediately available in front of you. Initial competition may occur within each domain of knowledge, such as color (red vs. green), size (large vs. small), and action (cut vs. pick). On the basis of the task instructions, top-down attentional influence will bias competition toward the color domain, and the winner from the color domain will receive a boost in its competitive weight and will subsequently exert inhibitory influence over other domains. In other words, in order to select the correct conceptual representation, the attentional system must bias selection toward the task-relevant domain and ignore information from task-irrelevant domains.

\section{What Are Some Factors That Affect Selection From Conceptual Representations?}

We will begin by considering a series of neuroimaging experiments reported by Thompson-Schill, D'Esposito, Aguirre, and Farah (1997), in which the authors manipulated selection demands in different ways across three semantic tasks - verb generation, object classification, and semantic comparison. In the verb generation task, subjects were presented with a noun and were asked to generate an associated verb as quickly as possible. Highselection items were nouns with many associated verbs and, importantly, with no prepotent verb response (e.g., rope-tie, hang, cut, or bound). On the other hand, lowselection items were nouns with few associated verbs or with a dominant verb response (e.g., scissors-cut). The critical manipulation was the number of competing verb responses.

In the object classification task, subjects were presented with a series of line drawings and were asked to classify each item on the basis of one of four attributes (e.g., big vs. small, light vs. heavy, manmade vs. natural, or expensive vs. cheap). Selection demands in this condition were high because the subjects had to focus on the relevant attribute of the object (e.g., size) and ignore other information that was irrelevant for that trial (e.g., weight). In the low-selection condition, the subjects were asked to verify whether a noun presented in fact named an accompanying picture. In this condition, selection demands were low because any activated information about the concept could be relevant to the decision; thus, there was no need to filter out information.

A similar manipulation was used in the semantic comparison task. In this task, the subjects were presented with a triad of words and were asked to decide which of the two probe words (e.g., tongue and bone) was more similar to a target word (e.g., tooth). In the high-selection condition, the subjects were instructed to make the comparison along a single dimension (e.g., color), and in the low-selection condition, the subjects were free to make a similarity judgment, using any criterion. Thus, in the high-selection condition, the subjects needed to selectively attend to a single attribute and ignore other, irrelevant information, whereas in the low-selection condition, the need to filter out irrelevant information was attenuated.

To evaluate the effect of selection demands, ThompsonSchill et al. (1997) directly compared the high-selection condition with the low-selection condition and found a similar pattern of activity across the experiments: Activity in the left inferior frontal gyrus (LIFG) was modulated by selection demands. These results suggest that the LIFG may be a candidate region for conceptual selection (see also Kan \& Thompson-Schill, 2004; ThompsonSchill, D'Esposito, \& Kan, 1999; Thompson-Schill et al., 1998). As has been described above, the lateral PFC has also been implicated in guiding behavior such as visual selective attention. Thus, one might naturally ask whether the same principles that apply to the control of visual selective attention have relevance to the study of conceptual selection.

These three selection manipulations can be described in terms of the biased competition model of visual selective attention. Consider the comparison of nouns hav- 
ing many associated verbs with those having few associated verbs: In terms of the biased competition model, this can be thought of as a manipulation of the initial competitive weight for each potential verb response. In the high-selection condition, initial competitive weights of the potential verb responses were assumed to be similar because each noun was not associated with any prepotent verb response. Therefore, the degree of competition was expected to be high. On the other hand, in the low-selection condition, one of the potential verb responses had a much higher initial competitive weight than the other verb responses did, and as such, the degree of competition would be low.

For the remainder of this review, we will consider factors that affect conceptual selection, borrowing terminology and distinctions from the literature on visual selective attention. For example, it may be useful to distinguish between top-down and bottom-up effects on conceptual selection. In this context, bottom-up influences refer to factors that are inherent to the stimuli themselves (e.g., number of competing verb responses), and top-down influences refer to factors that are manipulated by task instructions or recent retrieval history (e.g., type of similarity judgment).

Competition among conceptual representations may also come in two forms: within domain and across domain. (Later, we will return to the question of how to describe conceptual domains.) For example, in the comparison task, information about one attribute or domain (e.g., color) was assumed to compete with information from other domains (e.g., shape). Thus, in the high-selection condition, across-domain competition was hypothesized to be the source of increased selection demands. In contrast, in the verb generation task, competition could occur both within a domain and across different domains: The task of selecting a single verb response among a large set of weakly activated action representations (e.g., purr, scratch, or play) and also irrelevant but activated nonaction representations (e.g., dog) could involve both within-domain (i.e., action) and across-domain competition.

In our initial investigations into the process of conceptual selection, we did not distinguish between topdown and bottom-up influences on selection, nor did we systematically vary whether the source of competition was within domain or across domain. However, when these effects are considered in light of theories of visual selective attention, it seems likely that one or both of these distinctions will prove important in our understanding of conceptual selection. For example, our observation that patients with LIFG damage sometimes err on the verb generation task by failing to produce any response and, at other times, err by producing a nonverb response might be explained by a failure to inhibit either within-domain or across-domain competitors, respectively (Thompson-Schill et al., 1998). As we review the growing body of evidence for conceptual selection, we will attempt to integrate the conceptual selection literature with the visual selective attention literature.

\section{Phonological Competition}

To date, all of the studies in which the effects of phonological competition on word retrieval have been investigated have examined bottom-up effects on selection. For example, Desmond, Gabrieli, and Glover (1998) used a stem completion task to explore the relationship between LIFG activity and selection within the phonological domain. In a stem completion task, subjects were given a three-letter stem and were asked to generate as many words as possible that start with those three letters (e.g., sta__ ). The investigators manipulated the number of possible completions in two conditions (i.e., few vs. many). They argued that the number of possible completions was positively correlated with selection demands: Stems with more possible completions will activate more phonological competitors (e.g., stamp, stand, stack, or stallion) and will increase selection demands during word retrieval, when compared with stems with few possible completions (e.g., psa_; psalm). Indeed, Desmond and colleagues observed increased activity in the lateral PFC on trials with many completions, as compared with trials with few completions. Although the local maxima observed by Desmond and colleagues in the middle frontal gyrus is different from that observed by ThompsonSchill et al. (1997; Thompson-Schill et al., 1999), who reported local maxima in the inferior frontal gyrus, these findings are consistent with the general idea that activity in the lateral PFC is modulated by selection demands (see also Tippett, Gendall, Farah, \& Thompson-Schill, 2004, for convergent findings from patients with Alzheimer's disease).

\section{Lexical Competition}

During word retrieval, competition is not limited to phonological representations. Competition between lexical representations during word production has been investigated in a number of recent studies. For example, Robinson, Blair, and Cipolotti (1998) described an aphasic patient with lateral frontal damage whose impairments were best described with Luria's term dynamic aphasia; an extensive series of experiments indicated that the patient's ability to retrieve a word was affected by the number of competing responses. For example, when given a stem of a sentence and asked to generate a single word to complete it, the patient would fail with a sentence such as "Bob went to the store to buy some ...," although she would succeed with "Bob takes his coffee with milk and ..."

One task that has been used extensively to study the process of single-word retrieval - and the effects of competition thereon-is confrontation picture naming. Consider the animal depicted in Figure 6A. When one is asked to name that animal, the visual image may trigger many conceptual representations, and in order to select the appropriate representation, one must inhibit all other competitors. How is this task accomplished? In Levelt et al.'s (1991) model of picture naming, the authors proposed a two-stage model of lexical retrieval. According to 

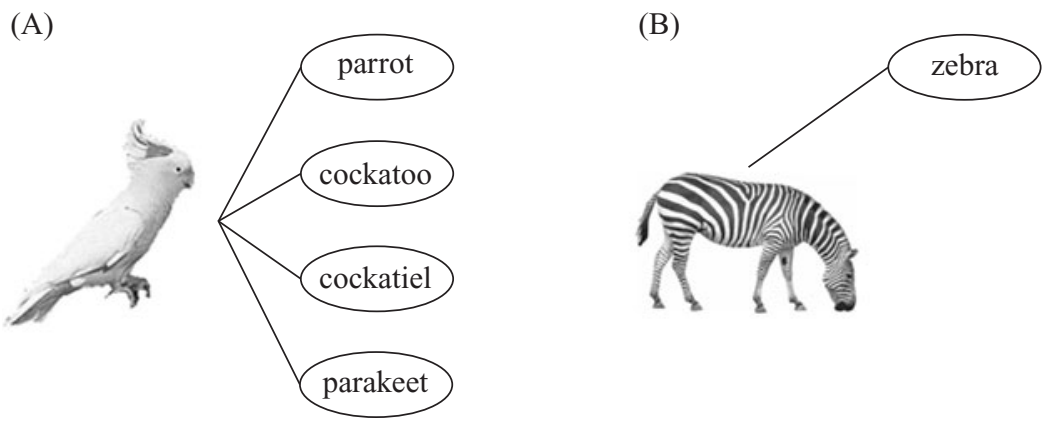

(C)

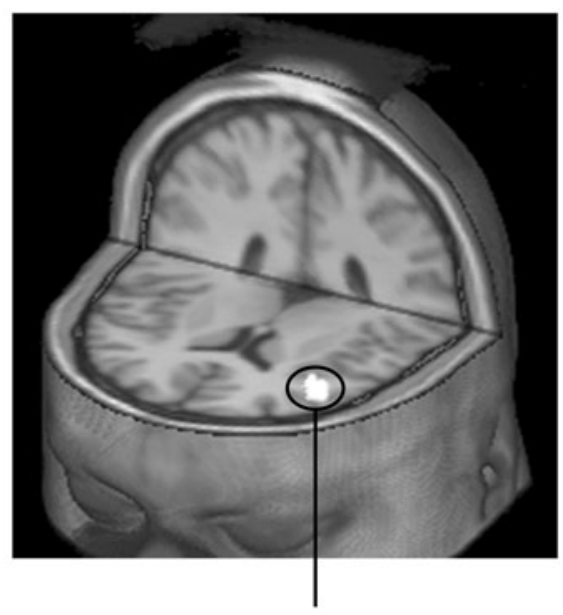

high selection $>$ low selection

Figure 6. Examples of (A) high-selection and (B) low-selection items in the context of picture naming. Panel $\mathrm{C}$ depicts greater activity in the left inferior frontal gyrus for the high-selection condition (low name agreement) than for the low-selection condition (high name agreement condition; Kan \& Thompson-Schill, 2004).

this model, an input concept is automatically generated upon perception of an object, and after perceptual analyses of the visual input, a set of semantically driven candidate lemmas become activated. These lemmas may include competing names for the same picture (e.g., parrot or cockatiel) and/or semantically related competitors (e.g., peacock or phoenix). A selection process then follows, from which only one of these alternatives will be selected, and this is the only item that is encoded phonologically and, ultimately, articulated as a response (see also Fraisse, 1969). Thus, selection demands are higher for items that elicit multiple representations than for those items that elicit only one representation (Figure 6B). However, the precise mechanism of selection was not discussed in Levelt et al.'s proposal.

In a behavioral experiment, Kremin, Hamerel, Dordain, De Wilde, and Perrier (2000) investigated the relationship between name agreement and picture-naming latency. Name agreement refers to the degree of concordance with which subjects name a given picture. For example, a picture with $76 \%$ name agreement means that only $76 \%$ of the subjects provided the same name for a given picture (e.g., parrot), and the other $24 \%$ gave alternative responses (e.g., cockatoo, parakeet, etc.). Kremin and colleagues proposed that the more competing alternatives there are, the longer it takes to select an appropriate response. Indeed, the authors reported an inverse correlation between name agreement and response latency.

These results may be understood in terms of the biased competition model as well, which differs from Levelt et al.'s (1991) model of selection as a separate stage in processing. Perhaps when a stimulus triggers multiple conceptual representations, those representations enter into a selection template. Bias is given to the conceptual representation that is most similar to the perceptual stimulus. Depending on the exact stimulus, various types of information, such as form and color, may enter into biasing the selection process. Converging evidence across different domains will generate a winner. Thus, the more conceptual representations triggered by a stimulus, the more competition is expected, and the longer it takes for a winner to emerge.

In a recent neuroimaging study, we proposed that selection among competing picture names is subserved by 
the LIFG (Kan \& Thompson-Schill, 2004). We tested this idea by varying selection demands as a function of picture name agreement. We proposed that the number of competing name alternatives evoked by a picture may modulate LIFG activity during picture naming, and we predicted higher LIFG activity in the low name agreement (high-selection) condition than in the high name agreement (low-selection) condition. Indeed, we found that the LIFG was sensitive to selection manipulation: Activity in the LIFG was greater in the high-selection condition than in the low-selection condition (see Figure 6).

Thus far, all of the effects we have discussed can be described as bottom-up influences on word retrieval: Both the number of lexical competitors activated by a picture and the number of phonological competitors activated by a word stem are inherent properties of the stimuli. However, lexical selection can also be affected by top-down influences, such as semantic context. For example, when one is asked to name a picture, the stimulus will trigger names that are associated with that object and also other concepts that are associated with the object. Therefore, other concepts that belong to the same semantic network are likely to compete with the pictured concept as well. To artificially increase the degree of competition, researchers have manipulated the strength of these competitors by varying the context in which a picture is to be named.

For example, Damian, Vigliocco, and Levelt (2001) reported that picture-naming latencies were longer when the pictures in a series were from the same semantic category (e.g., cow, sheep, goat, donkey, and pig) than when category membership of the pictures in the series was mixed (e.g., cow, bicycle, dress, scissors, and armchair). The authors interpreted these results in terms of competition among coactivated lexical entries (see Vigliocco, Vinson, Damian, \& Levelt, 2002, for similar findings). As is suggested by Levelt et al.'s (1991) model of picture naming, a cohort of semantic alternatives is automatically activated when a picture is encountered. The semanticblocking manipulation reinforces an overlapping set of semantic alternatives, thus increasing the competitive weight of each item. This semantic blocking phenomenon may parallel the local homogeneity effect discussed earlier: As homogeneity of distractors increases, search efficiency decreases.

A similar paradigm has been used to investigate picturenaming performance in a nonfluent aphasic patient with a suspected lesion in the anterior left hemisphere (Wilshire $\&$ McCarthy, 2002). Although the patient's standard picture-naming performance was preserved, his naming accuracy decreased dramatically when the stimuli were blocked by semantic category. This decrement in performance as a result of category blocking is similar to that reported in neurologically intact individuals, as was described above (Damian et al., 2001; Vigliocco et al., 2002). The authors attributed the patient's deficit to a selection problem. As was suggested earlier, the blocking manipulation increased the competitive weights of the competitors; whereas an intact selection mechanism will be slowed by the increased competition, an impaired selection mechanism may not be able to handle the demand at all, and thus, an increase in errors is expected.

\section{Semantic Competition}

During language comprehension, one must map a lexical representation onto a stable semantic representation; semantic competition occurs when multiple incompatible semantic representations are activated by a single lexical item. Although competition occurs to some extent during the processing of all words, certain words, such as polysemous words, provide an extreme case of heightened semantic competition. For example, the word pen can refer to either the writing implement or a small confined space for animals. In many cases, one meaning is more common (i.e., the dominant meaning) than the other (i.e., the secondary meaning). In addition to meaning dominance, context also affects which meaning is likely to be selected. For example, Simpson and Burgess (1985) suggested that when an ambiguous word is encountered, activation initially spreads to both word meanings, and initial activation strength is determined by meaning dominance. The second stage involves context integration. Consider the ambiguous word pen in the context of a sentence: The sentence "I went to the stationery store and bought a pen" will bias the writing implement meaning of the word, whereas the sentence "I spent a weekend at a farm and saw chickens in a pen" will bias the secondary meaning of the word. Thus, selection among competing word meanings is affected by both top-down (i.e., context) and bottom-up (i.e., meaning dominance) processes. Both of these effects can be understood in terms of the biased competition model: The initial competitive weight of the meaning will reflect the frequency (i.e., dominance) of that meaning, but attentional biases can modulate meaning selection to match the current context. These effects may have a parallel in the visual selective attention literature, such as Jonides and Gleitman's (1972) report of the effects of context on the interpretation of the ambiguous figure " $\mathrm{O}$ " (see also Hamm \& Taylor, 1997).

Numerous other studies have explored factors that affect selection of meaning with lexically ambiguous words (e.g., Milberg, Blumstein, \& Dworetzky, 1987; Sereno, 1995; Swaab, Baynes, \& Knight, 2002; Swinney, 1979; Van Petten \& Kutas, 1990); here, we focus on a few recent studies. Metzler (2001) used a semantic priming paradigm to investigate word meaning selection in patients with frontal damage. Subjects were presented with word triplets and were asked to read each word aloud. Each triplet consisted of a cue word, an ambiguous word, and a target word. The relationship among the three words was manipulated, and two different trial types were included: concordant and discordant. For example, the cue word (e.g., biology) would cue one meaning of the ambiguous word (e.g., cell), and the target word was either concordant with the cued meaning (e.g., tissue) or discordant with the cued meaning (e.g., prison). The ambiguous words used in the experiment always had two 
balanced meanings; that is, both meanings of the word would be equally likely to be retrieved if the ambiguous word were presented alone. The subjects' response time on the last word was recorded, and a priming effect was calculated on the basis of the response time difference between the concordant and the discordant conditions.

Metzler (2001) argued that if word meaning selection was accomplished successfully, a facilitation effect on response times on the last word would be expected in the concordant condition, when compared with the discordant condition. For example, with the triad biology-celltissue, reading the word biology would bias the meaning of cell toward the tissue meaning; therefore, subjects should be faster at reading the word tissue than the word prison. Even though prison is related to the jail meaning of the word cell, that meaning was selected against and was thus inhibited. Metzler found that whereas neurologically intact control subjects and patients with right frontal lesions benefited from the cue in the concordant condition, left frontal patients did not experience facilitation. Metzler interpreted the lack of facilitation as an indication that left frontal patients had problems selecting one of the two ambiguous meanings. Since both meanings were still active, no facilitation was observed.

Another possible explanation of these data, in terms of the biased competition model, is that since both meanings of the ambiguous words are balanced, the initial competitive weights are expected to be similar. In an intact system, competitive weight of each meaning is biased by the cue word, and thus there is increasing competitive weight for the concordant meaning and decreasing competitive weight for the discordant meaning. On the other hand, the biasing mechanism in an impaired system is ineffective at updating the competitive weights as a result of the cue word, and both meanings remain viable.

In addition to an item's long-term history (e.g., meaning dominance), recent retrieval history also exerts an influence over meaning selection, because recently retrieved items are more likely to have higher competitive weights than are recently inhibited items. In a series of behavioral experiments, Shivde and Anderson (2001) effectively reversed the competitive weights of the dominant and the secondary meanings of an ambiguous word by manipulating an item's recent retrieval history. This was accomplished by asking subjects to repeatedly retrieve the secondary meaning of a word.

During the initial exposure, the subjects were shown a series of word pairs and were asked to judge the relatedness of each word pair. For each ambiguous word, both meanings were shown (e.g., bank-money, the dominant meaning, and bank-river, the secondary meaning). In the second phase of the experiment, the subjects were given a cue and were asked to generate the second word of the pair (e.g., bank-r___ ). Feedback was provided initially to ensure that the subjects retrieved the correct word. After multiple retrieval of the secondary meaning, the subjects were given a cue to retrieve the dominant meaning of the word (e.g., bank-m ), and response times on those trials were measured. Shivde and Anderson (2001) reported a suppression effect (i.e., longer reaction time) of the dominant word meaning as a result of repeated retrieval of the secondary meaning of an ambiguous word. Furthermore, the magnitude of the suppression effect was scaled to the number of times the secondary meaning was retrieved. In terms of the biased competition model, repeatedly retrieving the same meaning of a word is likely to lead to an increase in the representation's competitive weight, which will lead to inhibition of the competitors. Thus, when asked to retrieve the recently suppressed meaning, the subjects' response times were lengthened.

\section{Summary}

In this section, we have presented evidence for the involvement of the lateral PFC in selection among conceptual representations. As we discussed with visual selective attention, there are both bottom-up and top-down influences on conceptual selection. Both types of competition seem to be well accounted for by the biased competition model.

\section{GENERAL DISCUSSION}

In this article, we have considered selection mechanisms that operate in two different domains: visual selective attention and selection among conceptual representations, with the former focusing on selecting relevant information from the external world and the latter dealing with selection among internal conceptual representations. We have also attempted to discuss many of the phenomena observed in the latter case of selection in terms of the biased competition model (Desimone \& Duncan, 1995), which was initially formulated to explain mechanisms involved in visual selective attention.

Furthermore, we have proposed that the lateral PFC may be a candidate region for the mechanism involved in conceptual selection. Much like visual selective attention, the role of the lateral PFC is to bias selection toward task-relevant information and to exert inhibitory control over task-irrelevant information. Given the considerable evidence linking semantic retrieval and the left temporal lobe (Hodges, Graham, \& Patterson, 1995; Hodges, Patterson, Oxbury, \& Funnell, 1992; see Saffran, 2000, and Thompson-Schill, 2003, for reviews), it is conceivable that the lateral PFC and the left temporal lobe work in concert during selection from conceptual representations. The contributions that each of these regions make to conceptual selection may parallel the division of labor observed in studies of visual selective attention, where it has been reported that extrastriate visual areas resolve within-domain competition but association cortices (frontal and parietal) are recruited in cases of across-domain competition.

This raises one of several questions that emerge from this comparison of visual selective attention and conceptual selection: What are the domains of long-term 
conceptual representations? (For competing answers to this question, see Allport, 1985, and Caramazza \& Shelton, 1998.) When discussing across-domain competition in the case of a semantic comparison task, we equated domains with attributes of knowledge (e.g., color). However, without a formal model of the long-term representations of conceptual information, it is difficult to establish which types of competition should be considered within domain and which should be considered across domain. This remains a question for future investigation.

The attentional template is perhaps one of the most powerful components of the biased competition model (Desimone \& Duncan, 1995). The attentional template is formulated as a flexible template that can accommodate any goal-relevant information that will enhance selection. It is this flexibility that facilitates our adaptive behavior. The attentional template may be capable of representing more than stimulus properties, such as color and shape, and may include information such as word meaning in the case of conceptual selection. However, the format in which the attentional template is represented is underspecified. Is the format propositional, depictive, or both? Or is the format also dependent on the task at hand, so that one type of stimulus (e.g., words) will elicit one format (i.e., propositional) and another type of stimulus (e.g., pictures) will elicit another format (i.e., depictive)? Answers to these questions may provide further insight into the interaction between conceptual and perceptual representations.

As was alluded to earlier, another open question concerns the extent to which the distinction between perceptual and conceptual representations should be viewed as a dichotomy or as two ends of a continuum. There are a number of potentially relevant differences between the two that could affect the manner in which competition is reconciled during the process of selection. For example, given the transient nature of sensory input from the external world, relative to the enduring nature of long-term knowledge structures, one might expect the decay function of activation to be quite different between these two types of representations. The implications of these types of differences for models such as the biased competition model - and the extent to which the differences are best described in quantitative or qualitative terms - will have to be resolved as theories of conceptual selection are formalized.

Finally, throughout this review, we have been purposefully vague about the localization of a putative attentionalbiasing mechanism in the frontal cortex for the sake of simplicity in this first-pass comparison. Although there is substantial evidence that both visual selective attention and conceptual selection recruit PFC, no studies have directly examined the colocalization of these processes, in the same group of subjects. Even if one could argue that the same computational mechanism accounts for selection of both perceptual and conceptual representations and further demonstrate that this mechanism was subserved by the same brain region in both cases, there could be functional specializations within the PFC that reflect the domain - perceptual or conceptual-in which selection is being biased. Notions of domainspecific heterogeneity within areas of functional homogeneity echo some of the ideas most strongly championed by Pat Goldman-Rakic during her career. Answers to this question, along with some of the others raised throughout this review, will move us closer to an understanding of frontal lobe function that Goldman-Rakic devoted her life to pursuing.

\section{REFERENCES}

Allport, D. A. (1985). Distributed memory, modular subsystems and dysphasia. In S. K. Newman \& R. Epstein (Eds.), Current perspectives in dysphasia (pp. 207-244). Edinburgh: Churchill Livingstone.

Barch, D. M., Braver, T. S., Sabb, F. W., \& Noll, D. C. (2000). Anterior cingulate and the monitoring of response conflict: Evidence from an fMRI study of overt verb generation. Journal of Cognitive Neuroscience, 12, 298-309.

Botvinick, M., BRAVer, T. S., Barch, D. M., Carter, C. S., \& Cohen, J. D. (2001). Conflict monitoring and cognitive control. Psychological Review, 108, 624-652.

Buchel, C., Josephs, O., Rees, G., Turner, R., Frith, C. D., \& FrisTON, K. J. (1998). The functional anatomy of attention to visual motion: A functional MRI study. Brain, 121, 1281-1294.

Bundesen, C. (1990). A theory of visual attention. Psychological Review, 97, 523-547.

BUNDESEN, C. (1996). Formal models of visual attention: A tutorial review. In A. F. Kramer \& M. G. H. Coles (Eds.), Converging operations in the study of visual selective attention (pp. 1-43). Washington, DC: American Psychological Association.

Caramazza, A., \& Shelton, J. R. (1998). Domain-specific knowledge systems in the brain: The animate-inanimate distinction. Journal of Cognitive Neuroscience, 10, 1-34.

Chawla, D., ReEs, G., \& FrIston, K. J. (1999). The physiological basis of attentional modulation in extrastriate visual areas. Nature Neuroscience, 2, 671-676.

Damian, M. F., Vigliocco, G., \& Levelt, W. J. (2001). Effects of semantic context in the naming of pictures and words. Cognition, 81, B77-B86.

Desimone, R., \& Duncan, J. (1995). Neural mechanisms of selective visual attention. Annual Review of Neuroscience, 18, 193-222.

Desmond, J. E., GaBRIELI, J. D., \& Glover, G. H. (1998). Dissociation of frontal and cerebellar activity in a cognitive task: Evidence for a distinction between selection and search. NeuroImage, 7, 368-376.

Driver, J., \& Frackowiak, R. S. (2001). Neurobiological measures of human selective attention. Neuropsychologia, 39, 1257-1262.

DunCan, J., \& Owen, A. M. (2000). Common regions of the human frontal lobe recruited by diverse cognitive demands. Trends in Neurosciences, 23, 475-483.

Egeth, H. E., Virzi, R. A., \& Garbart, H. (1984). Searching for conjunctively defined targets. Journal of Experimental Psychology: Human Perception \& Performance, 10, 32-39.

FARAH, M. J. (1989). Semantic and perceptual priming: How similar are the underlying mechanisms? Journal of Experimental Psychology: Human Perception \& Performance, 15, 188-194.

FletCher, P. C., \& Henson, R. N. A. (2001). Frontal lobes and human memory: Insights from functional neuroimaging. Brain, 124, 849881.

Folk, C. L., \& Remington, R. [W.] (1999). Can new objects override attentional control settings? Perception \& Psychophysics, 61, 727-739.

Folk, C. L., Remington, R. W., \& Johnston, J. C. (1992). Involuntary covert orienting is contingent on attentional control settings. Journal of Experimental Psychology: Human Perception \& Performance, $\mathbf{1 8}$, 1030-1044

Folk, C. L., Remington, R. W., \& Wright, J. H. (1994). The structure of attentional control: Contingent attentional capture by apparent motion, abrupt onset, and color. Journal of Experimental Psychology: Human Perception \& Performance, 20, 317-329. 
Fraisse, P. (1969). Why is naming longer than reading? Acta Psychologica, 30, 96-103.

FUSTER, J. M. (1973). Unit activity in prefrontal cortex during delayedresponse performance: Neuronal correlates of transient memory. Journal of Neurophysiology, 36, 61-78.

Fuster, J. M., \& AlEXANDER, G. E. (1971). Neuron activity related to short-term memory. Science, 173, 652-654.

GaZZANIGA, M. S., IVRY, R., \& MANGUN, G. R. (2002). Cognitive neuroscience: The biology of the mind. New York: Norton.

GoldMAN-RAKIC, P. S. (1987). Circuitry of primate prefrontal cortex and regulation of behavior by representational memory. In F. Plum (Ed.), Handbook of physiology: The nervous system (pp. 373-417). Bethesda, MD: American Physiological Society.

Green, B., \& ANderson, L. K. (1956). Color coding in a visual search task. Journal of Experimental Psychology, 51, 19-24.

HAMm, J., \& TAYLOR, T. L. (1997). Category effects in temporal visual search. Canadian Journal of Experimental Psychology, 51, 36-46.

Hodges, J. R., Graham, N., \& Patterson, K. (1995). Charting the progression in semantic dementia: Implications for the organisation of semantic memory. Memory, 3, 463-495.

Hodges, J. R., Patterson, K., OXXury, S., \& Funnell, E. (1992). Semantic dementia: Progressive fluent aphasia with temporal lobe atrophy. Brain, 115, 1783-1806.

JACOBSEN, C. F. (1935). Functions of frontal association areas in primates. Archives of Neurology \& Psychiatry, 33, 558-560.

JaCobsen, C. F. (1936). Studies of cerebral function in primates. Comparative Psychology Monographs, 13, 1-68.

Jonides, J., \& Gleitman, H. (1972). A conceptual category effect in visual search: $\mathrm{O}$ as letter or as digit. Perception \& Psychophysics, 12, 457-460.

Kahneman, D. (1973). Attention and effort. Englewood Cliffs, NJ: Prentice-Hall.

Kan, I. P., \& Thompson-Schill, S. L. (2004). Effect of name agreement on prefrontal activity during overt and covert picture naming. Cognitive, Affective, \& Behavioral Neuroscience, 4, 43-57.

Kastner, S., De Weerd, P., Desimone, R., \& UnGerleider, L. G. (1998). Mechanisms of directed attention in the human extrastriate cortex as revealed by functional MRI. Science, 282, 108-111.

Kastner, S., De WeERD, P., Pinsk, M. A., Elizondo, M. I., Desimone, R., \& UNGERLEIDER, L. G. (2001). Modulation of sensory suppression: Implications for receptive field sizes in the human visual cortex. Journal of Neurophysiology, 86, 1398-1411.

Kastner, S., Pinsk, M., De WeERD, P., Desimone, R., \& Ungerleider, L. G. (1999). Increased activity in human visual cortex during directed attention in the absence of visual stimulation. Neuron, 22, 751761.

Kastner, S., \& Ungerleider, L. G. (2000). Mechanisms of visual attention in the human cortex. Annual Review of Neuroscience, 23, 315-341.

KASTNER, S., \& UngerLeider, L. G. (2001). The neural basis of biased competition in human visual cortex. Neuropsychologia, 39, $1263-$ 1276.

Kremin, H., Hamerel, M., Dordain, M., De Wilde, M., \& Perrier, D. (2000). Age of acquisition and name agreement as predictors of mean response latencies in picture naming of French adults. Brain \& $\mathrm{Cog}_{-}$ nition, 43, 286-291.

Levelt, W. J. M., Schriefers, H., Vorberg, D., Meyer, A. S., PechMANN, T., \& HAVINGA, J. (1991). The time course of lexical access in speech production: A study of picture naming. Psychological Review, 98, $122-142$.

LoGAN, G. D. (2004). Cumulative progress in formal theories of attention. Annual Review of Psychology, 55, 207-234.

Luck, S. J., \& Vecera, S. P. (2002). Attention: From tasks to mechanisms. In S. Yantis (Ed.), Stevens' handbook of experimental psychology: Vol. 1. Sensation and perception (pp. 235-286). New York: Wiley.

Metzler, C. (2001). Effects of left frontal lesions on the selection of context-appropriate meanings. Neuropsychology, 15, 315-328.

Milberg, W., Blumstein, S. E., \& DworetzKy, B. (1987). Processing of lexical ambiguities in aphasia. Brain \& Language, 31, 138-150.

Milham, M. P., Banich, M. T., WebB, A., Barad, V., Cohen, N. J.,
WszaleK, T., \& Kramer, A. F. (2001). The relative involvement of anterior cingulate and prefrontal cortex in attentional control depends on nature of conflict. Cognitive Brain Research, 12, 467-473.

Miller, E. K., \& CohEN, J. D. (2001). An integrative theory of prefrontal cortex function. Annual Review of Neuroscience, 24, 167-202.

Miller, J. (1989). The control of attention by abrupt visual onsets and offsets. Perception \& Psychophysics, 45, 567-571.

Moran, J., \& DESIMONE, R. (1985). Selective attention gates visual processing in the extrastriate cortex. Science, 229, 782-784.

Nelson, J. K., Reuter-Lorenz, P. A., Sylvester, C.-Y. C., Jonides, J., \& Sмiтн, E. E. (2003). Dissociable neural mechanisms underlying response-based and familiarity-based conflict in working memory. Proceedings of the National Academy of Sciences, 100, 11171-11175.

O'Craven, K. M., Rosen, B. R., Kwong, K. K., Treisman, A. M., \& SAVOY, R. L. (1997). Voluntary attention modulates fMRI activity in human MT-MST. Neuron, 18, 591-598.

PASHLER, H., \& HARRIS, C. R. (2001). Spontaneous allocation of visual attention: Dominant role of uniqueness. Psychonomic Bulletin \& Review, 8,747-752.

PESSOA, L., KASTNER, S., \& UNGERLEIDER, L. G. (2003). Neuroimaging studies of attention: From modulation of sensory processing to topdown control. Journal of Neuroscience, 23, 3990-3998.

Robinson, G., Blair, J., \& CipolotTi, L. (1998). Dynamic aphasia: An inability to select between competing verbal responses? Brain, 121(Pt. 1), 77-89.

SAFFRAN, E. M. (2000). The organization of semantic memory: In support of a distributed model. Brain \& Language, 71, 204-212.

Schumacher, E. H., \& Jiang, Y. (2003). Neural mechanisms for response selection: Representation specific or modality independent? Journal of Cognitive Neuroscience, 15, 1077-1079.

SERENO, S. C. (1995). Resolution of lexical ambiguity: Evidence from an eye movement priming paradigm. Journal of Experimental Psychology: Learning, Memory, \& Cognition, 21, 582-595.

SHIVDE, G., \& ANDERSON, M. C. (2001). The role of inhibition in meaning selection: Insights from retrieval-induced forgetting. In D. S. Gorfein (Ed.), On the consequences of meaning selection: Perspectives on resolving lexical ambiguity (pp. 175-190). Washington, DC: American Psychological Association.

Simpson, G. B., \& Burgess, C. (1985). Activation and selection processes in the recognition of ambiguous words. Journal of Experimental Psychology: Human Perception \& Performance, 11, 28-39.

STALLER, J. D. (1982). Word superiority in word detection. \& Psychophysics, 31, 237-242.

STROOP, J. R. (1935). Studies of interference in serial verbal reactions. Journal of Experimental Psychology, 18, 643-662.

SWAAB, T. Y., BAYNES, K., \& KNIGHT, R. T. (2002). Separable effects of priming and imageability on word processing: An ERP study. Cognitive Brain Research, 15, 99-103.

SwINNEY, D. A. (1979). Lexical access during sentence comprehension: (Re)consideration of context effects. Journal of Verbal Learning \& Verbal Behavior, 18, 25-37.

Sylvester, C.-Y. C., Wager, T. D., Lacey, S. C., Hernandez, L., Nichols, T. E., SMith, E. E., \& Jonides, J. (2003). Switching attention and resolving interference: fMRI measures of executive functions. Neuropsychologia, 41, 357-370.

ThOMPSON-SCHILL, S. L. (2003). Neuroimaging studies of semantic memory: Inferring "how" from “where." Neuropsychologia, 41, 280292.

Thompson-Schill, S. L., D’Esposito, M., Aguirre, G. K., \& Farah, M. J. (1997). Role of left inferior prefrontal cortex in retrieval of semantic knowledge: A reevaluation. Proceedings of the National Academy of Sciences, 94, 14792-14797.

THOMPSON-SCHILL, S. L., D’EsPosito, M., \& KAN, I. P. (1999). Effects of repetition and competition on prefrontal activity during word generation. Neuron, 23, 513-522.

Thompson-Schill, S. L., Swick, D., Farah, M. J., D'Esposito, M., KAN, I. P., \& KNIGHT, R. T. (1998). Verb generation in patients with focal frontal lesions: A neuropsychological test of neuroimaging findings. Proceedings of the National Academy of Sciences, 95, 15855-15860.

TippetT, L. J., Gendall, A., Farah, M. J., \& Thompson-Schill, S. L. 
(2004). Selection ability in Alzheimer's disease: Investigation of a component of semantic processing. Neuropsychology, 18, 163-173. Treisman, A. M., \& Gelade, G. (1980). A feature integration theory of attention. Cognitive Psychology, 12, 97-136.

Treisman, A. M., \& Gormican, S. (1988). Feature analysis in early vision: Evidence from search asymmetries. Psychological Review, $\mathbf{9 5}$, $15-48$.

Van Petten, C., \& Kutas, M. (1990). Interactions between sentence context and word frequency in event-related brain potentials. Memory \& Cognition, 18, 380-393.

van Veen, V., Cohen, J. D., Botvinick, M. M., Stenger, V. A., \& CARTER, C. S. (2001). Anterior cingulate cortex, conflict monitoring, and levels of processing. NeuroImage, 14, 1302-1308.
Vigliocco, G., Vinson, D. P., Damian, M. F., \& Levelt, W. J. (2002). Semantic distance effects on object and action naming. Cognition, 85, B61-B69.

WiLshire, C. E., \& McCARThy, R. A. (2002). Evidence for a contextsensitive word retrieval disorder in a case of nonfluent aphasia. Cognitive Neuropsychology, 19, 165-186.

YANTIS, S., \& JoNIDES, J. (1984). Abrupt visual onsets and selective attention: Evidence from visual search. Journal of Experimental Psychology: Human Perception \& Performance, 10, 601-621.

(Manuscript received August 8, 2004; revision accepted for publication October 14, 2004.) 